\title{
Ionic Polymerization in Ionic Liquids
}

\author{
Yibo Wu \\ Additional information is available at the end of the chapter
}

http://dx.doi.org/10.5772/intechopen.77183

\begin{abstract}
Ionic liquids have emerged as a new class of solvents for ionic polymerization due to their low volatility, chemical stability, high conductivity, wide electrochemical window. The advantages and limitations of application of ionic liquids as solvents for ionic polymerization processes are critically discussed in this chapter. The field of cationic polymerization in ionic liquid has undergone rapid growth in recent years. The most important types of cationic monomers, such as styrene and its derivatives, vinyl ethers and isobutylene have been polymerized in ionic liquids; even undergo living polymerization. Corresponding elementary reactions of cationic polymerization in ionic liquids were proposed. Methyl methacrylate and styrene can undergo anionic polymerization in ionic liquids. However, ionic liquids seem unsuitable solvents for anionic polymerization.
\end{abstract}

Keywords: ionic liquid, cationic polymerization, anionic polymerization, elementary reactions, ionic environment

\section{Introduction}

Ionic liquids are organic salts, and their physical and chemical properties can be fine-tuned by selection of the cation and anion. The most significant properties of ionic liquids are their negligible vapor pressure. So, ionic liquids have been recognized as green solvents alternative to volatile organic solvents. Application of ionic liquids in chemical processes has blossomed within the last decade. Although radical polymerization, electrochemical polymerization, and polycondensation in ionic liquids have been investigated by many researchers, there has been little study on the application of ionic liquids in ionic polymerization. Ionic liquids are regarded as highly polar but non-coordinating solvents, enabling them as ideal solvents for ionic polymerization. 
Ionic polymerization is referred to as a classsic cationic when the active terminal group is positively charged, or as a pseudocationic if this group forms the positive end of active dipole. By the same token we refer to ionic polymerization as a classic anionic when the charge of the active group is negative, or as a pseudoanionic when the active group forms the negative end of an active dipole. Whenever electrically charged end-groups are formed, suitable counterions have to be present in the polymerizing system to ensure its electric neutrality. So, the counterions chaperon the growing active to form ion-pairs during the initiation and propagation reaction. These ion-pairs usually exist in three thermodynamically distinct forms, which are referred to as tight ion-pairs, loose ion-pairs and free ion-pairs, and plays an important role for determination of polymerization characteristic. For example, in cationic polymerization, if this counterion is too nucleophilic, it will attack the carbenium ion to form a covalently bond, which in principle does not initiate to cationic monomers. So, the ion environment of ionic liquid must affect balance of ion pairs owing to the high polarity and high charge density, and then influence polymerization process.

Moreover, properties of ionic liquids such as melting point, polarity, viscosity, and solubility of monomers, which can be fine-tuned by the adequate selection of the cation and anion constituents, also significantly affect the feasibility and regularity of ionic polymerization. High polarity of ionic liquid contributes to stabilize the cationic active center, but it also exhibits the low solubility for cationic monomers. Thus, desirable ionic liquids hope to achieve polar and non-polar balance scale, which can provide a moderately polar environment to solubilize cationic monomers and a polar environment to stabilize carbocation. In this chapter, the mechanism of polymerization, advantages and limitations of application of ionic liquids as solvents for ionic polymerization processes are critically discussed.

\section{Cationic polymerization in ionic liquids}

Cationic polymerization is an important technique to produce (co)polymers with predictable molecular weight and monomer sequence. Especially, living/controlled cationic polymerization represents an attractive technique for the synthesis of well-defined polymers, such as telechelic, star polymer, graft copolymer, etc. Industrialization products, such as bromide functionalized poly(isobutylene-co-p-methylstyrene) random copolymer, butyl rubber and poly (styrene- $b$-isobutylene- $b$-styrene) thermoplastic elastomer, play an important role in the tire industry, coronary stent, chewing gum and other fields. However, during the preparation of these products by traditional cationic polymerization, halogenated alkane (e.g., methyl chloride) usually used as solvents and cause environmental pollution due to its toxicity, volatile and corrosively. So it should be replace by green solvents. In addition, various Lewis acids (e.g., $\mathrm{BF}_{3}, \mathrm{SnCl}_{4}, \mathrm{TiCl}_{4}, \mathrm{AlCl}_{3} \mathrm{OBu}_{2}$, and $\mathrm{AlCl}_{3}$ ) were used as coinitiator in cationic polymerization. It is difficult to separate these Lewis acid catalysts from the reaction products, and reuse/ disposal of these catalysts is also a big challenge to industry.

Ionic liquid is regarded as the ideal medium of cationic polymerization, which can be recycled and no pollution to the environment. So, applications of ionic liquids in cationic 
polymerizations have great theoretical and practical significance for the development of environment-friendly and low-energy consumption. The most important monomers polymerizing through cationic mechanism are styrene and its derivatives, vinyl ethers and isobutylene (IB). The commonness of these cationic monomers is that the active species can be stabilized by the substituents on the olefinic group. And the way of stabilization of the corresponding carbocation are shown in Table 1 [1]. We discuss cationic polymerization of these monomers in ionic liquids respectively in following sections.

\subsection{Styrene and its derivatives}

Styrene is a well-known, commercially available vinyl monomer that undergoes polymerization via cationic as well as radical, anionic, and coordination pathways. More recently, developed air- and water-stable neutral ionic liquids (for instance, 1-butyl-3-methylimidazoliumhexafluorophospate ([BMIM][PF$]$ ) [2-4] and trihexyltetradecylphosphonium bis(trifluoromethanesulfonyl)amide $\left.\left(\left[\mathrm{P}_{6,6,6,14}\right]\left[\mathrm{NTf}_{2}\right]\right)\right)$ [5] have been applied in cationic polymerization. Moreover, cationic polymerization of styrene in N-butyl-N-methylpyrrolidiniumbis-(trifluoromethanesulfonyl) amide $\left(\left[\mathrm{P}_{14}\right]\left[\mathrm{NTf}_{2}\right]\right)[6]$ ionic liquid with organoborate acids as initiators indicates some living/ controlled characteristics.

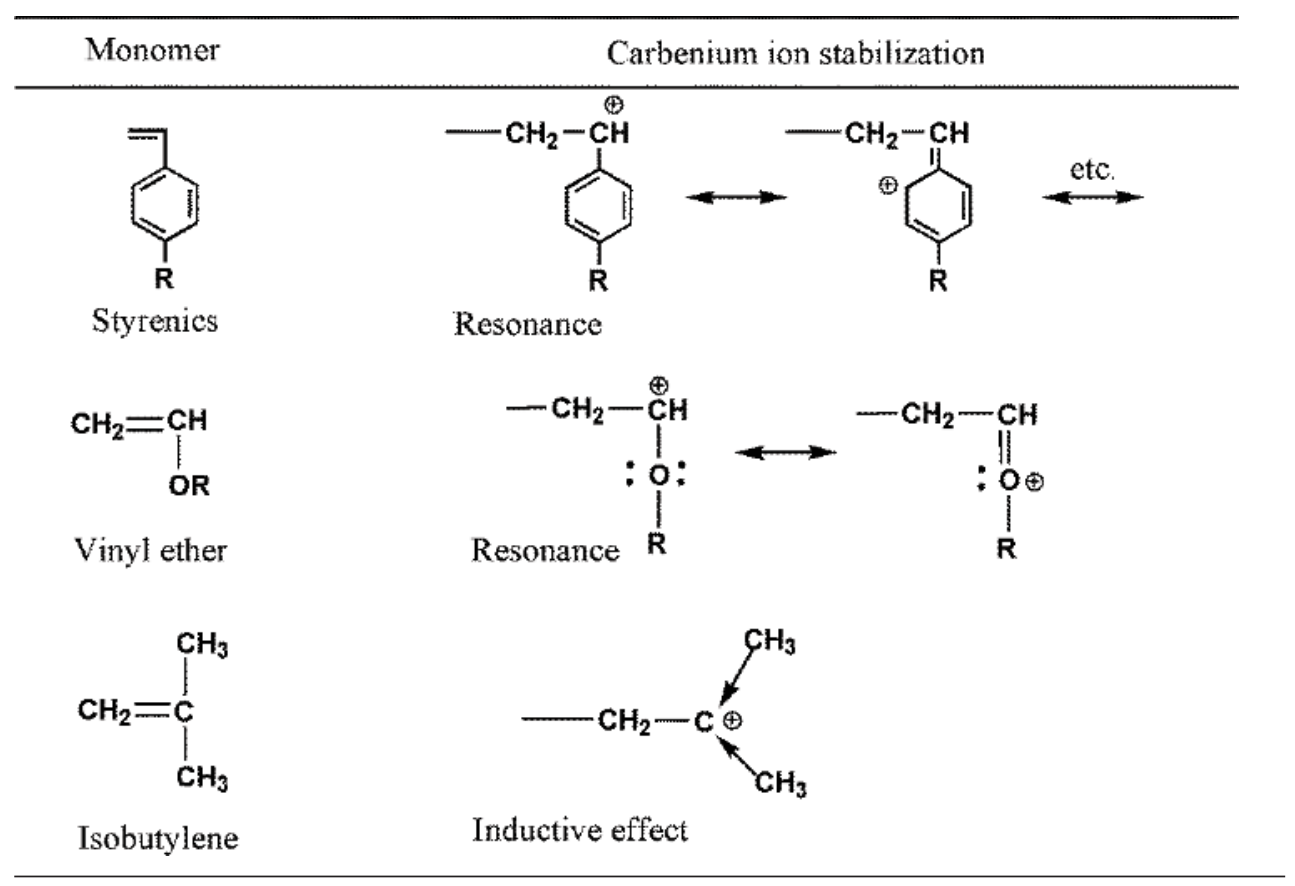

Table 1. Cationic monomers and stabilization of the corresponding active species. 
In the traditional cationic polymerization of styrene, the lack of strongly electron-donating groups renders the growing carbocation unstable and thus results in side reactions, such as chain transfer accompanied by $\beta$ proton elimination and Friedel-Crafts alkylation on the phenyl ring of the monomer unit. However, the polymerization mechanism of styrene and its derivatives cationic polymerizations in ionic liquids is still vague.

Usually, the low solubilities of the monomers in ionic liquids impede the efficiency of these polymerization reactions. In order to search for suitable ionic liquids for styrene and its derivatives, we firstly screened and selected target ionic liquids by quantum chemically based computations (the COSMO-RS method) [7]. The COSMO-RS method was successfully used to screen potential ionic liquids as solvents with respect to the solubility of $p$-methylstyrene used in cationic polymerization (Figure 1). We also have demonstrated that COSMO-RS is a valuable tool for the preliminary screening of solvents for cationic monomers without the need for extensive experimental data. The guiding principle of the selection of suitable ionic liquids for use in cationic polymerization was obtained from COSMO-RS calculations. The monomer solubilities in ionic liquids are highly dependent on physical and chemical properties which determine by anion and cation in ionic liquid. Larger nonpolar regions of the cation or anion in ionic liquids result in higher monomer solubility for cationic monomers, for example, longer alkyl chains of the cation or anion contributes to higher $p$-methylstyrene solubilities in imidazolium-based ionic liquids.

It is instructive to compare the organic solvents and ionic liquids in cationic polymerizations. So, we comprehensively compared the cationic polymerizations of styrene [8] and $p$-methylstyrene [9] in ionic liquids with those in organic solvents employing a series of

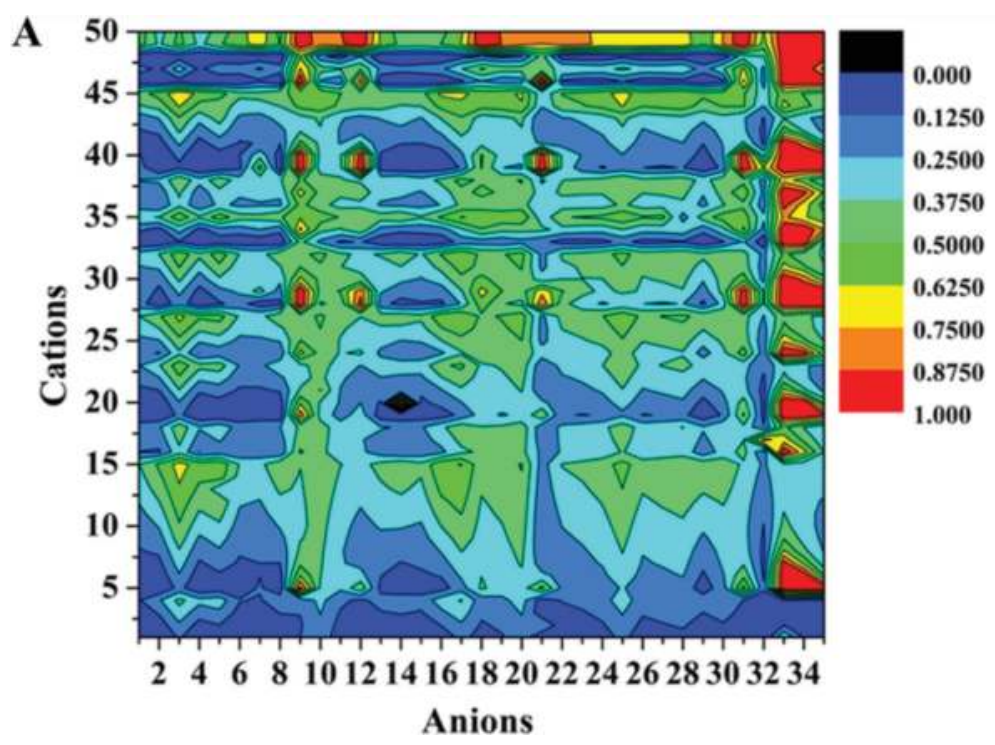

Figure 1. Predicted solubilities of $p$-methylstyrene in 1750 types (50 cations $\times 35$ anions) of ionic liquids. 
initiating systems. Considering that the effect of polymerization by the initiator, styrene cationic polymerization initiated by water exhibited lower yield and lower $M_{n}$ compared with a,a-dimethylbenzyl chloride and 2-chloro-2,4,4-trimethylpentane. It was speculated that the low initiating efficiency might occur by the formation of hydrogen bonding for water in the imidazole-based ionic liquid. Water is more likely to initiate cationic polymerization in organic solvents.

The polymerization rate of styrene and $p$-methylstyrene in ionic liquids rely mainly on solvent polarity and viscosity. We found that initial polymerization rate of styrene in ionic liquid was similar to that in dichloromethane which resulting from interactions between viscosity and polarity factors of ionic liquids. The $[\mathrm{bmim}]\left[\mathrm{PF}_{6}\right]$ ionic liquid had a considerably higher normalized solvent polarity $\mathrm{E}^{\mathrm{N}}{ }_{\mathrm{T}}$ as compared to dichloromethane. On the one hand, the high the polarity of solvent give rise to the faster the reaction rate in cationic polymerization; on the other hand, the high viscosity of ionic liquid reduces the monomer diffusion and thus slow down the rate of polymerization. So, the polymerization rate in ionic liquids was similar to that in dichloromethane, which was a consequence of viscosity and polarity of ionic liquid.

Styrene and $p$-methylstyrene cationic polymerizations proceeded in a milder exothermic manner in ionic liquids than in traditional organic solvents. The milder reactions in ionic liquid may be due to the relatively higher heat capacity of the ionic liquid. The relatively higher heat capacity of ionic liquid could absorb more heat during the cationic polymerization.

On basis of terminal structure and kinetics of polymerization, we proposed the corresponding elementary reactions of styrene and $p$-methylstyrene cationic polymerization in ionic liquids, as shown in Figures 1 and 2, respectively.

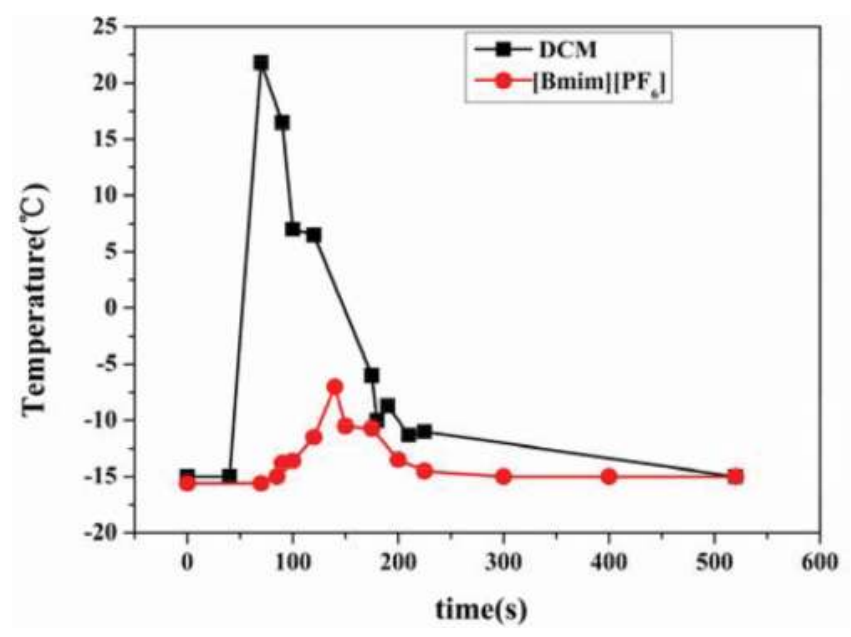

Figure 2. Temperature changes vs. time plots for cationic polymerization of styrene with $\mathrm{CumCl} /$ Lewis acid initiating system in ionic liquid and dichloromethane at $-15^{\circ} \mathrm{C}$ operating temperature, (black square) dichloromethane, (red circle) $[\mathrm{Bmim}]\left[\mathrm{PF}_{6}\right]$. 
In initiation reactions, ion-pairs of carbocation and counterion were first formed by complexation reactions between coinitiator Lewis acid and initiator. Comparing with cation of ionic liquid, the carbocation in ion-pairs was easier to attack the styrene or $p$-methylstyrene to initiate cationic polymerization. Also, because of anion of ionic liquid was very weakly nucleophilic species, the counterion of ion-pairs was more closely to carbocation and made them approachable to interact with growing active center. Therefore, we assumed that at least one portion of chain termination reactions directly took place toward counterion rather than anion of ionic liquid. So, the anions or cations of the ionic liquids did not participate in any elementary reactions in the whole cationic polymerization. Despite all this, the anion of ionic liquid could stabilize the propagating carbocation active by dispersing the charge of carbocation. But it was still insufficient to stabilize the propagating carbocation to achieve a controlled/living cationic polymerization.

The terminal structure of polystyrenes analyzed by ${ }^{1} \mathrm{H}-\mathrm{NMR}$ spectroscopy and MALDI-TOF spectra which clearly indicated that main chain termination reactions in ionic liquid directly took place toward halide-based counterion or toward Friedel-Crafts reaction, rather than $\beta$-hydrogen elimination reaction (Figure 3).

The sterical hindrance of counteranion influenced the insertion of monomer molecules into the propagating carbocation. Thus, the interaction between propagating carbocation and

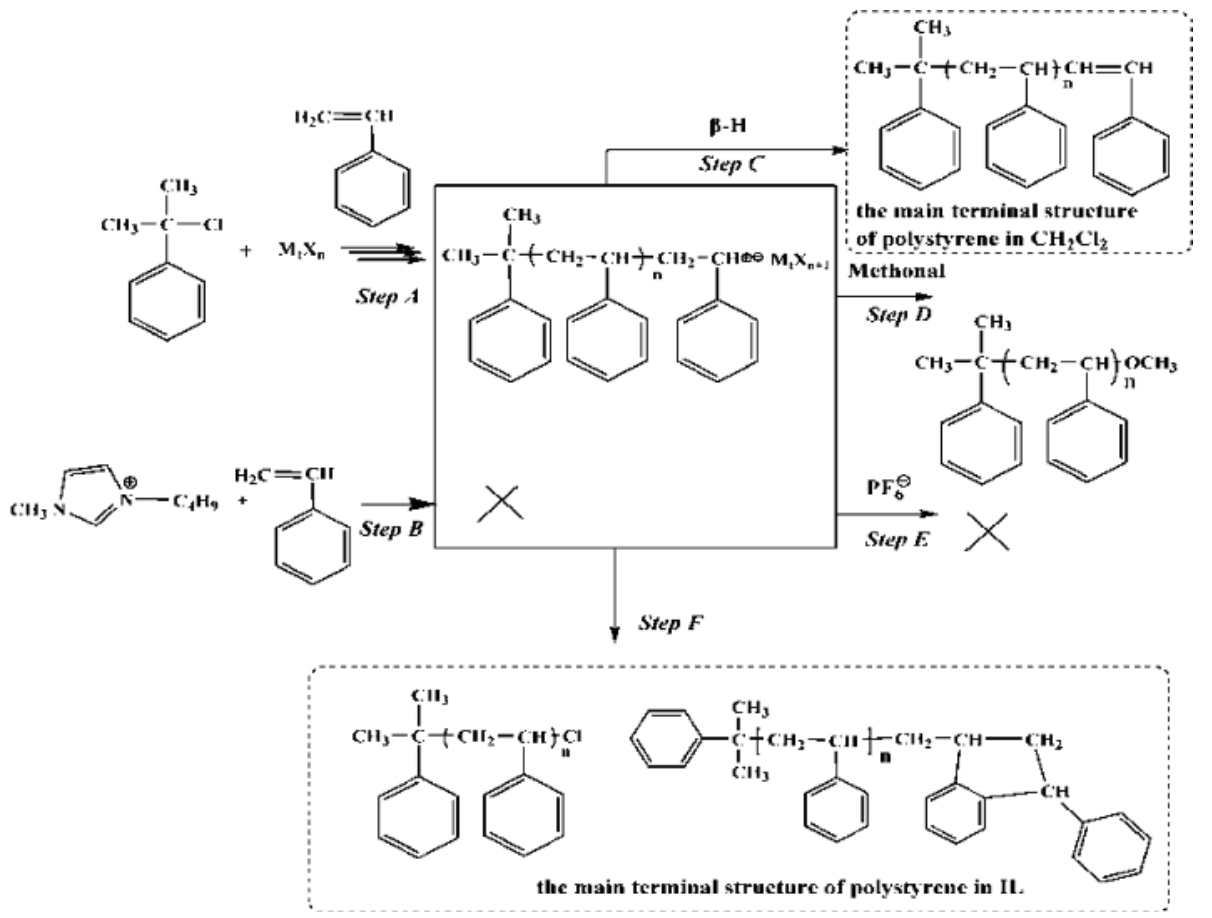

Figure 3. Polymerization pathway of cationic polymerization of styrene with CumCl/Lewis acid in $[\mathrm{bmim}]\left[\mathrm{PF}_{6}\right]$. 
metal halide-based counterion was directly responsible for stereoregulation. Due to the existence interaction between growing carbocation with anion of ionic liquid, the interaction between the growing carbocation and counteranion become weaker. So, sterical hindrance of counteranion was reduced in ionic liquid which led to lower stereoregulation (Figure 4).

MacFarlane [5] has successfully demonstrated that the a controlled cationic polymerization of styrene has been achieved in $\left[\mathrm{C}_{4} \mathrm{mpyr}\right]\left[\mathrm{NTf}_{2}\right]$ ionic liquid using bis(oxalato)boric acid $(\mathrm{HBOB})$ as initiators. The hydrophobic nature of the $\left[\mathrm{C}_{4} \mathrm{mpyr}\right]\left[\mathrm{NTf}_{2}\right]$ ionic liquid allowed relatively easy to achieve styrene cationic polymerization. The molecular weights of polystyrenes obtained in $\left[\mathrm{C}_{4} \mathrm{mpyr}\right]\left[\mathrm{NTf}_{2}\right]$ ionic liquid increased with decreasing $\mathrm{HBOB}$ concentration with narrow polydispersity and were predominantly syndiotactic. In another study cationic polymerization of styrene initiated with $\mathrm{AlCl}_{3}$ in ionic liquid ([bmim] $\left[\mathrm{PF}_{6}\right]$ ), supercritical $\mathrm{CO}_{2}$ and organic solvent $\left(\mathrm{CH}_{2} \mathrm{Cl}_{2}\right)$ was investigated [4]. The only conclusion was that polymerization rates and molecular weights were higher than in organic solvent.

\subsection{Vinyl ethers}

The cationic polymerization of vinyl ethers under "non-living " conditions, has been known for many years and been used commercially [10]. These polymerizations are characterized by extremely high polymerization rates and the occurrence of chain transfer and termination reactions with the formation of different kinds of unsaturated end-groups. In additional, vinyl ethers are among the most reactive monomers in conventional (dry conditions) cationic polymerization, even more reactive than pMOS. So, chain transfer reaction is more likely to occur in vinyl ethers cationic polymerization. Low temperatures are usually employed in an attempt to reduce side reactions that destroy the propagating centers. In order to meet

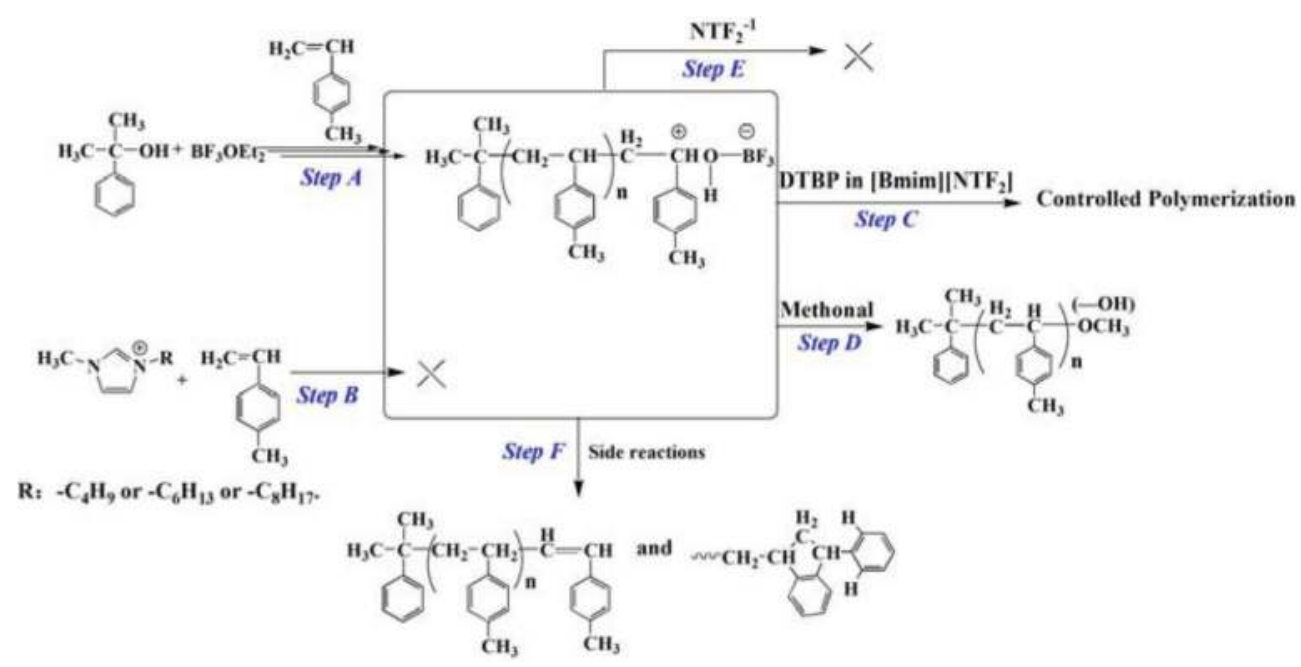

Figure 4. Proposed mechanism for the cationic polymerization of $p$-methylstyrene with the $\mathrm{CumOH} / \mathrm{BF}_{3} \mathrm{OEt}_{2}$ initiating system in $\mathrm{NTf}_{2}^{-1}$ based ionic liquids. 
the requirements of low temperature for vinyl ethers cationic polymerizations, we mainly focused on the low-melting-point ionic liquids, such as $[\mathrm{omim}]\left[\mathrm{BF}_{4}\right]$ ionic liquid.

In order to further understand the ion environment and its effect on cationic polymerization, we compared the characteristics of IBVE cationic polymerization in organic molecule medium. Table 2 showed the data of IBVE polymerization in dichloromethane in the same condition as $\left[\right.$ omim] $\left[\mathrm{BF}_{4}\right]$. Comparing with those obtained in ionic liquid, the yields of poly(IBVE)s obtained in organic molecule medium were lower $(\sim 30 \%)$. These indicated that IBVE cationic polymerizations were more likely to participate chain transfer and chain termination reactions in organic molecule medium. In addition, The $M_{n}$ of poly(IBVE)s obtained in ionic liquid were much higher than that in organic molecule medium. The ionic liquid had a considerably higher normalized solvent polarity $\mathrm{E}_{\mathrm{T}}^{\mathrm{N}}$ as compared to organic molecule medium, such as dichloromethane. Usually, the higher the polarity of solvent gives in cationic polymerization, the higher the $M_{n}$ of poly(IBVE) obtained.

Like that of styrene, the cationic polymerization of IBVE in dichloromethane system proceeded in a highly exothermic manner. For example, in $\mathrm{IBVE}-\mathrm{HCl} / \mathrm{TiCl}_{4}$ initiating system, the exothermic peak reached to $24^{\circ} \mathrm{C}$. However, the exothermic peak in [omim] $\left[\mathrm{BF}_{4}\right]$ ionic liquid reached to $9^{\circ} \mathrm{C}$ with $\sim 90 \%$ monomer conversion. The temperature rise and fall periods were slower than that in dichloromethane. IBVE cationic polymerization in ionic liquid proceed in mild exothermic reactions may be due to the relatively higher viscosity and higher heat capacity of the ionic liquids. The high viscosity could slow down the reaction rate; the relatively higher heat capacity could absorb more heat during the catonic polymerization.

From reaction kinetics analysis, we found that the first-order plots of $\ln ([\mathrm{M} 0] /[\mathrm{M}])$ vs. time were not linear for isobutyl vinyl ether (IBVE) cationic polymerization in [omim] $\left[\mathrm{BF}_{4}\right]$ ionic liquid. Analyzing from end microstructure, the side reactions took place by chain-breaking via predominant $\beta$-proton elimination from $-\mathrm{CH}_{2}-$ in the growing carbocation and then by protic reinitiation to create a new polymer chain, resulting in the formation of polymer chains with exo-olefin terminal group. Once 2,6-di-tert-butylpyridine was used; $\beta$-proton had been trapped. Thus, the polymerization in ionic liquid exhibited some characteristics of a living/ controlled process (Figure 5).

\begin{tabular}{llllllll}
\hline Entry & Solvent & Coinitiator & Time (min) & Conv. (\%) & $\mathbf{M n}$ & $\mathbf{M w}$ & Mw/Mn \\
\hline 1 & Dichloromethane & $\mathrm{Al}_{2} \mathrm{Et}_{3} \mathrm{Cl}_{3}$ & 1.5 & 20 & 21,880 & 40,700 & 1.86 \\
2 & Dichloromethane & $\mathrm{SnCl}_{4}$ & 2.5 & 27 & 16,490 & 31,500 & 1.91 \\
3 & Dichloromethane & $\mathrm{TiCl}_{4}$ & 2.5 & 29 & 13,200 & 24,000 & 1.82 \\
4 & {$\left[\right.$ omim] $\left[\mathrm{BF}_{4}\right]$} & $\mathrm{Al}_{2} \mathrm{Et}_{3} \mathrm{Cl}_{3}$ & 10.0 & 82 & 38,010 & 65,380 & 1.72 \\
5 & {$\left[\right.$ omim] $\left[\mathrm{BF}_{4}\right]$} & $\mathrm{SnCl}_{4}$ & 15.0 & 76 & 21,070 & 38,140 & 1.81 \\
6 & {$[\mathrm{omim}]\left[\mathrm{BF}_{4}\right]$} & $\mathrm{TiCl}_{4}$ & 15.0 & 83 & 18,140 & 36,600 & 2.02 \\
\hline
\end{tabular}

Conditions: Initiator: $[\mathrm{IBVE}-\mathrm{HCl}]=0.003 \mathrm{M},[\mathrm{IBVE}]=1.04 \mathrm{M}$, the molar ratio of coinitiator to $\mathrm{IBVE}-\mathrm{HCl}=16, \mathrm{~T}=0^{\circ} \mathrm{C}$; $\mathrm{Mn}($ theor $)=34,300 \mathrm{~g} / \mathrm{mol}$.

Table 2. Cationic polymerizations of IBVE using various coinitiators in ionic liquid and dichloromethane. 


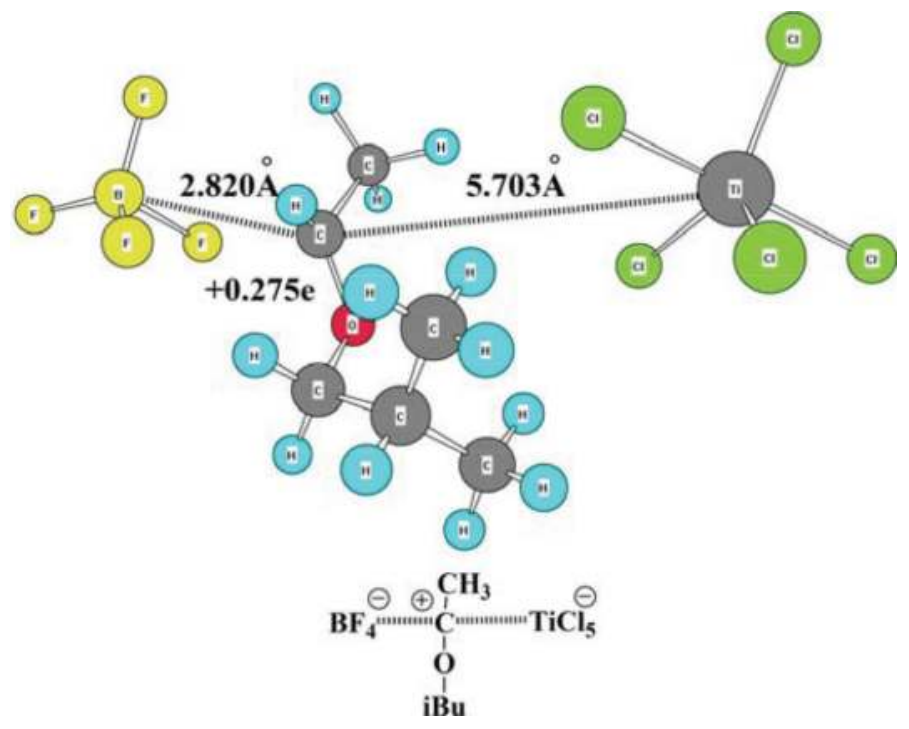

Figure 5. The optimized geometries of metal halide-based counterion, propagating carbocation and anion of ionic liquid.

The long-lived species were observed in monomer addition experiments. The [omim] $\left.\mathrm{BF}_{4}\right]$ ionic liquid did not participate elementary reactions during IBVE cationic polymerization. However, [omim] $\left[\mathrm{BF}_{4}\right]$ should affect not only the stability of active center but also the interaction between propagating carbocation and counterion. We want to understand what was the cause of production of the long-lived species. The density functional theory was used to study the interactions among propagating active center, counterion and ionic liquid.

According to the geometry, the propagating carbocations of the poly(IBVE)s in [omim][BF4] interacted with not only metal halide-based counterions, but also soft Lewis basic $\mathrm{BF}_{4}$ anions. It was also noted that the charge on the propagating carbocation in ionic liquid was separated by its interaction with anion of ionic liquid leading to form relative stabilized propagating carbocation. However, these interactions were still insufficient to stabilize the propagating carbocation to achieving a controlled polymerization.

\subsection{Isobutylene}

Among cationic monomers, isobutylene is no doubt the most extensively studied one as it polymerizes only by cationic mechanism. In comparison with silicones or polyphosphazenes, polyisobutylene-based products exhibit such unique properties as chemical resistance, low permeability, good thermal and oxidative stability, mechanical dampening. The high- $\left(M_{\mathrm{n}}>120,000 \mathrm{~g} / \mathrm{mol}\right)$, medium- $\left(M_{\mathrm{n}}=40,000-100,000 \mathrm{~g} / \mathrm{mol}\right)$ and, low-molecular weight $\left(M_{\mathrm{n}}<5000 \mathrm{~g} / \mathrm{mol}\right)$ polyisobutylenes (PIB) have been achieved commercial success and were widely used in the world.

Low molecular weight polyisobutylene possessing an exo-olefin terminal group (so-called highly reactive polyisobutylene, HR PIB) is a key intermediate in the manufacturing of 
motor oil and fuel additives, with worldwide production in excess of 750,000 tons per year. The cationic polymerizations of HR PIB in ionic liquids were described only in a patent literatures [11-14] and only limited information was available. The use of acidic chloroaluminate ionic liquids as catalysts for low molecular weight polyisobutylene $\left(M_{\mathrm{n}}=1000 \mathrm{~g} / \mathrm{mol}\right)$ in n-heptane should be particularly noteworthy. But, unfortunately, little information about the molecular weight distribution and microstructure of polyisobutylene oligomers was available. Recently, Kostjuk [15] has present new catalysts for the synthesis of HR PIB based on the combination of a chloroaluminate ionic liquids and diisopropyl ether, which allow to synthesize PIBs with a high content of exo-olefin end groups $(\geq 90 \%)$ and relatively narrow MWD (Mw/Mn $\leq 2.0)$ (Figure 6).

Indeed, we did not find suitable ionic liquids for isobutylene cationic polymerization to synthesize the high-, medium- $M_{n}$ polyisobutylene. Generally, isobutylene is hard to dissolve in ionic liquids. The number of possible combinations of anions and cations in ionic liquids are very numerous. The properties of ionic liquids can be fine-tuned by selection of the cation and anion. It is difficult to discuss their properties in general because their properties depend on the structure of cation and anion. So, in order to screen the potential neutral ionic liquid solvent for isobutylene cationic polymerization, we used density functional theory calculations to investigate the inter-ionic interactions of ionic liquids and the interactions of ionic liquids with isobutylene [16]. The geometry was explained by the change of total energy, inter-molecular distance and ESP charge. The most stable gas-phase structures of ion pairs (IPs) and IPs-IB indicated that hydrogen bonding with the C2-hydrogen on the imidazole ring played a dominating role in the formation of IPs. The addition of IB did not change the dominant interactions of IPs. Compared with previous literature, the dissolution mechanism of IB in ionic liquids is that IB molecules occupy the free space of the cavities which are primarily created by small angular rearrangements of the anions. The potential solvent for IB polymerization is the ionic liquid with weaker interactions of anion and ion pair with IB. This work was motivated by the selection of ionic liquids as polymerization solvents. This study will also provide a broad range for future studies on cationic polymerizations in ionic liquids.
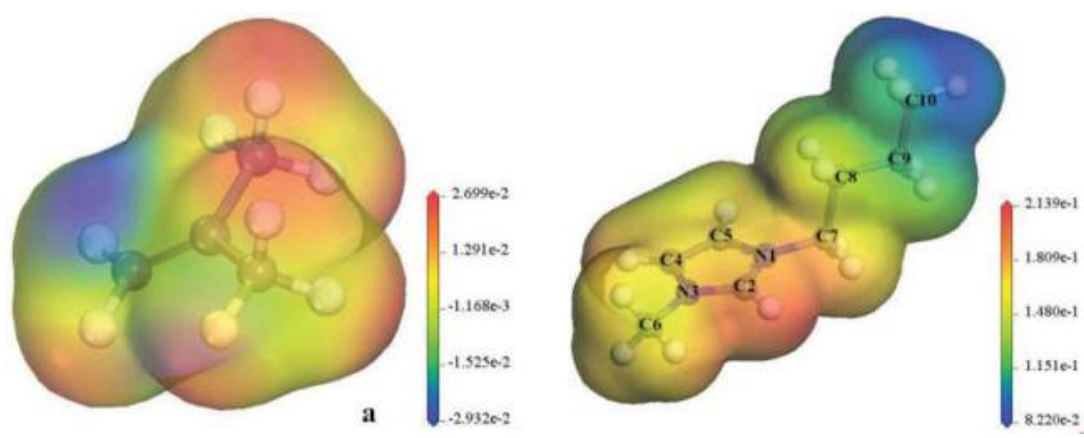

Figure 6. The electrostatic potentials (ESP) surface of isobutylene (IB, a) and [Bmim] + (b). 


\section{Anionic polymerization in ionic liquids}

\subsection{Methyl methacrylate}

Generally, anionic polymerizations need strict dehydration and oxygen-free conditions. Ionic liquids can be easy to dry under vacuum at high temperature due to their negligible volatility $[17,18]$. So, cumbersome handling procedures for conventional volatile solvent such as distillation in the presence of drying agents was no need for ionic liquid. In order to understand mechanism and characteristics of anionic polymerization in ionic liquids, the methyl methacrylate (MMA) anionic polymerizations were carried out in ionic liquids by using alkyl lithium initiators such as n-butyllithium (n-BuLi) and diphenylhexyl lithium (DPHLi). The results were compared with those obtained for polymerization reactions in conventional solvents such as tetrahydrofuran (THF) and toluene. The MMA anionic polymerization in [NTf $\left.{ }_{2}\right]$ based ionic liquids did not yield polymers because the initiator were be deactivated by attacking on the trifluoromethyl group. However, as compared with in tetrahydrofuran, the MMA anionic polymerization proceeded in $\left[\mathrm{C}_{4} \mathrm{mim}\right]\left[\mathrm{PF}_{6}\right]$ ionic liquid with lower monomer conversion. The reaction between the initiator and the imidazolium cation and high polymerization temperature resulted in lower yields. Analyzing from the ${ }^{1} \mathrm{H}-\mathrm{NMR}$ spectrum, the alkyl lithium initiator withdrawn hydrogen atom at the imidazolium ring, which was the main cause of deactivating initiator. The tacticity of PMMA initiating by DPHLi in $\left[\mathrm{C}_{4} \mathrm{mim}\right]\left[\mathrm{PF}_{6}\right]$ was rich in $\mathrm{mm}$ triads. These results indicated that the propagation carbocation of PMMA in $\left[\mathrm{C}_{4} \mathrm{mim}\right]$ $\left[\mathrm{PF}_{6}\right]$ has a similar terminal structure with that in toluene.

Another side-reaction in anionic MMA polymerization initiated by alkyl lithium initiators was observed by Kubisa [19]. Analyzing the terminal structure by MALDI-TOF, it was found that chain transfers to ionic liquids were easy to occur at the early stages of propagation polymerization. The chain transfer reaction in ionic liquid is shown in Figure 7. Although MMA anionic polymerization put a limit on molecular weights $(\mathrm{Mn}<2000)$ in ionic liquid, all the PMMAs contained ionic end-groups derived from ionic liquids, which should be pay enough attention.
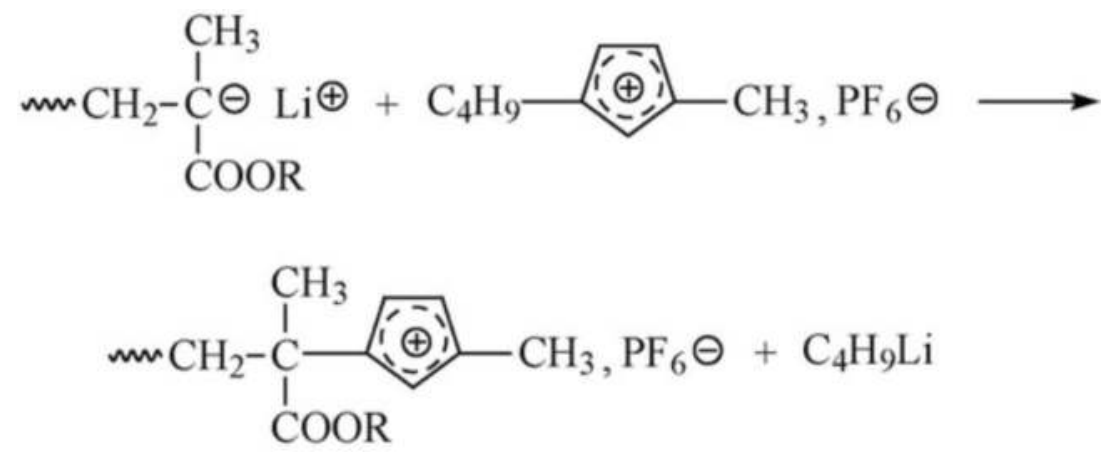

Figure 7. Chain transfer to ionic liquid in anionic polymerization of methyl methacrylate. 


\subsection{Styrene}

Styrene anionic polymerization initiated by butyllithium (BuLi) or sodium acetate (NaAc) in phosphonium-based ionic liquids has been reported by MacFarlane [20]. Although relatively high initiator concentration was used, polystyrene yield was still lower (10 20\%). Yields could be improved by addition of butyl imidazolium butane sulfonate zwitterion. Moreover, molecular weights kept to high (up to 400,000) with molecular weight distribution in the range of 1.4-2.1. This indicated that phosphonium-based ionic liquids were more suitable as solvents for styrene anionic polymerization than imidazolium-based ionic liquids.

\section{Conclusion}

In recent years, an enormous progress has been made in the development of ionic polymerizations in ionic liquid. The polymerization reactions for cationic and anionic monomers were realized in ionic liquids. Some cationic polymerizations in ionic liquid indicated some living/ controlled characteristics. However, only the control of molecular weight and polydispersity have been achieved. Telechelic polymers, macromonomers, block copolymers, polymer networks, star-shaped polymers have not been described in ionic liquids. So, in development of mechanism of living cationic polymerization, scientists need to strengthen scientific research further. Ionic liquids seem unsuitable solvents for anionic polymerization. Is it possible to find suitable ionic liquids for anionic polymerization by "designer-solvents"? The further theoretical studies are needed and the guiding roles of the relevant theories should be brought into full play.

\section{Acknowledgements}

This work was supported by the National Science Foundation of China (51573020), Beijing Natural Science Foundation (2172022).

\section{Conflict of interest}

The authors report no conflicts of interest in this work.

\section{Author details}

Yibo $\mathrm{Wu}$

Address all correspondence to: wuyibo@bipt.edu.cn

Department of Material Science and Engineering, Beijing Key Lab of Special Elastomeric Composite Materials, Beijing Institute of Petrochemical Technology, Beijing, China 


\section{References}

[1] Goethals EJ, Prez FD. Carbocationic polymerizations. Progress in Polymer Science. 2007; 32(2):220-246. DOI: 10.1016/j.progpolymsci.2007.01.001

[2] Biedroñ T, Kubisa P. Cationic polymerization of styrene in a neutral ionic liquid. Journal of Polymer Science, Part A: Polymer Chemistry. 2004;42:3230-3235. DOI: 10.1002/pola. 20158

[3] Baœko M, Biedroñ T, Kubisa P. Polymerization processes in ionic liquids. Cationic polymerization of styrene. Macromolecular Symposia. 2006;240:107-113. DOI: 10.1002/ masy.200650814

[4] Bueno C, Cabral V, Cardozo-Filho L, Dias M, Antunes O. Cationic polymerization of styrene in scCO2 and [bmim][PF6]. Journal of Supercritical Fluids. 2009;48:183-187. DOI: 10.1016/j.supflu.2008.09.023

[5] Vijayaraghavan R, MacFarlane D. Organoborate acids as initiators for cationic polymerization of styrene in an ionic liquid medium. Macromolecules. 2007;40:6515-6520. DOI: 10.1021/ma070668z

[6] Vijayaraghavan R, Macfarlane DR. Living cationic polymerisation of styrene in an ionic liquid. Chemical Communications. 2004;6:700-701. DOI: 10.1039/b315100j

[7] Zhang XQ, Guo WL, Wu YB, Li W, Li SX, Shang YW, Zhang JH. Solubility of monomers for chain polymerization in ionic liquids predicted by the conductor-like screening model for real solvents. Industrial and Engineering Chemistry Research. 2017;56:1469414703. DOI: $10.1021 /$ acs.iecr.7b04235

[8] Han L, Wu YB, Yang D, Wang H, Zhang XQ, Wei XL, Guo WL, Li SX. Characteristics and mechanism of styrene cationic polymerization in 1-butyl-3-methylimidazolium hexafluorophosphate ionic liquid. RSC Advances. 2016;6:105322-105330. DOI: 10.1039/ C6RA22284F

[9] Zhang XQ, Guo WL, Wu YB, Gong LF, Li W, Li XN, Li SX, Shang YW, Yang D, Wang $\mathrm{H}$. Cationic polymerization of p-methylstyrenein selected ionic liquids and polymerizationmechanism. Polymer Chemistry. 2016;7:5099-5112. DOI: 10.1039/c6py00796a

[10] Wu YB, Han L, Zhang XQ, Mao J, Gong LF, Guo WL, Gu K, Li SX. Cationic polymerization of isobutyl vinyl ether in an imidazole-based ionic liquid: Characteristics and mechanism. Polymer Chemistry. 2015;6:2560-2568. DOI: 10.1039/C4PY01784F

[11] Ambler PW, Hodgson PKG, Stewart NJ. (BP Chem. Ltd) EP, 05581871993

[12] Abdul-Sada A, Ambler PW, Hodgson PKG, Seddon KR, Stewart NJ. (BP Chem. Ltd) WO, 9521871. 1995

[13] Abdul-Sada A, Seddon KP, Stewart NJ. (BP Chem. Ltd) WO, 9521872. 1995

[14] Murphy V. (Symyx Tech. Inc.) WO, 00/32685. 2000 
[15] Vasilenko IV, Berezianko IA, Shiman DI, Kostjuk SV. New catalysts for the synthesis of highly reactive polyisobutylene: Chloroaluminate imidazole-based ionic liquids in the presence of diisopropyl ether. Polymer Chemistry. 2016;7:5615-5619. DOI: 10.1039/ c6py01325b

[16] Li XN, Guo WL, Wu YB, Li W, Gong LF, Zhang XQ, Li SX, Shang YW, Yang D, Wang $\mathrm{H}$. Investigation of the interactions between 1-butyl-3-methylimidazolium-based ionic liquids and isobutylene using density functional theory. Journal of Molecular Modeling. 2018;24:83. DOI: 10.1007/s00894-018-3586-y

[17] Kubisa P. Ionic liquids as solvents for polymerization processes-progress and challenges. Progress in Polymer Science. 2009;34:1333-1347. DOI: 10.1016/j.progpolymsci.2009.09.001

[18] Kokubo H, Watanabe M. Anionic polymerization of methyl methacrylate in an ionic liquid. Polymers for Advanced Technologies. 2008;19:1441-1444. DOI: 10.1002/pat.1210

[19] Biedron T, Kubisa P. Chain transfer to ionic liquid in an anionic polymerization of methyl methacrylate. Journal of Polymer Science Part A: Polymer Chemistry. 2007;45:4168-4172. DOI: $10.1002 /$ pola.22256

[20] Vijayaraghavan R, Pringle JM, MacFarlane DR. Anionic polymerization of styrene in ionic liquids. European Polymer Journal. 2008;44(6):1758-1762. DOI: 10.1016/ j.eurpolymj.2008. 02.028 